\title{
Analysis of Estimation Parameter for Energy Efficient in Wireless Sensor Network
}

\author{
Supriya Sarkar \\ School of Information Technology, UTD, RGPV \\ Bhopal, Madhya Pradesh-462033, India
}

\author{
Sanjeev Sharma \\ School of Information Technology, UTD, RGPV \\ Bhopal, Madhya Pradesh-462033, India
}

\begin{abstract}
In wireless sensor network energy fully depend on battery lifetime. Energy saving by making intelligent importance driven decision about message transmission in self organized method. The selective forwarder is a method which makes intelligent importance driven decision for saving energy. The development of a selective forwarder method based on decision theory which was not stable for network as well as before failure the route, how can recognized the failure route no one knows the idea, for that proposed an approach to develop optimal energy-aware transmission schemes by adaptive selective forwarder for quality of information as well as for stable network. However, in the work include stochastic process and routing protocol depends on parameters like available energy on a node, retransmission a message and message importance. By using these three parameters find out the best performance in network with analysis and comparison of adaptive selective forwarder and local-global forwarder method. The constant threshold values apply for saving energy of each node from source node to sink node for optimal solution in the network. The main focus of work is an analysis the estimated parameter which are used for getting efficient energy according to changing the level of energy of sensor node for increasing the lifetime of battery as well as increasing the lifetime of the network. Also calculate battery lifetime by using Markov decision process rule with proper network design.
\end{abstract}

\section{Keywords}

Wireless sensor network (WSN), adaptive selective forwarder, AODV protocol, Markov decision process (MDP).

\section{INTRODUCTION}

In a last decade wireless sensor network (WSN) going to be researcher interested area. Sensor network is distributed autonomous network for small and monitor the environment and cooperatively pass data through the network to the main location. It has enabled the development of low-cost, lowpower, and energy-constrained sensors and small in size for communicating within short distance. For designing of sensor network no need engineer, it is randomly allowed to access to a disaster relief operation. The wide range of area where sensor network is used given in [1] such as military, environment monitoring, health, home and agriculture etc. In many applications, it is objectionable or impractible to replace or recharge sensors. So that, the network lifetime becomes a critical concern in the design of WSN.

\subsection{Challenges in Wireless Sensor Network}

$[2]$

- Limited hardware means each node has limited processing and communication capabilities, and limited energy supply and bandwidth.

- $\quad$ Limited support for networking which the network is peer-to-peer with a mesh topology, dynamic, mobile, and unreliable connectivity.

- Limited support for software development means the tasks are typically real-time and massively distributed, involve dynamic collaboration among nodes, and must handle multiple competing events.

- $\quad$ Energy (Power consumption) means the sensor node fully depend on battery lifetime and by power of the battery is decide the network lifetime,

- Robustness and Scalability are vital for designing the sensor network.

\subsection{Routing Protocol}

While various energy efficient protocols have been proposed to prolong the network lifetime. There are different routing protocols as we saw in the traditional adhoc network, some of that used in wireless sensor network for efficient routing performance in the network such as AODV, DSR, TORA and DSDV [2]. Basically AODV protocols are used for getting high performance for network which is given in [3].The difference between wireless sensor network and traditional adhoc network was given in [4]

a) Sensor node is densely deployed.

b) Sensor node is prone to failure

c) Sensor node mainly uses for broadcast communication paradigm.

d) The topology of sensor network changes is frequented.

e) Sensor nodes are limited for power computation.

The energy efficient aware protocols are used in different ways for increasing the network lifetime. The energy consumption is the primary factor for wireless sensor network. For practically scenario sensor node batteries cannot be refilled, this node has a finite lifetime.

Energy in WSN can be saved by making intelligent importance driven decision about message transmission in self organized, different method applied for power management and efficient energy for increase batteries lifetime which given in [5]. 


\subsection{Markov Decision Process}

The Markov decision process is a stochastic tool used for mathematical formulation was given in [6][7]. A Markov decision Process is a stochastic process on the random variables of state $x_{t}$, action at, and rewards $r_{t}$, as given by the dynamic network. The process is defined by the conditional probabilities

$$
\begin{array}{cl}
P\left(x_{t}+1 \mid a_{t} ; x_{t}\right) & \text { Transition probability } \\
P\left(r_{t} \mid a_{t} ; x_{t}\right) & \text { Reward probability } \\
P\left(a_{t} \mid x_{t}\right)=\left(a_{t} \mid x_{t}\right) & \text { policy }
\end{array}
$$

The problem of optimal decision control which given in MDP to compute an optimal policy $\pi^{*}$ when the $\operatorname{model} P(x 0 \mid a: x)$, the rewards $P(r \mid a ; x)$, and the discount factor $\gamma \in R$ are known.

\subsection{Selective Forwarder Method}

Selective forwarding mechanism shows the node changes dynamically mean routed node from source node and send the energy level with packet to the neighbor node in case any node failure, then the packet transmitted to neighboring node but not energy. [8][9] Advantage of selective forwarded method such as:

- Intelligent decision making

- Less overhead signaling

- Maximizing packet distribution

- Self contained and adaptive scheme

\subsection{Success Index}

There are 3 technique involved in success index such as Zero index, Local index, global index. The zero index occur when take any transmission successful means in any case value must be 1 .The local index occur when transmitted node has no way to know if the message arrives to the sink but not happened in global index, the local index value is 1 if a neighboring node forwards the message otherwise zero. The global index occurs when the message must travel through several nodes before arriving to destination, the message transmitted successfully means value is 1 in case of transmitting messages to sink otherwise zero. This success index used for finding out the total reward as well as reward technique. This technique indicates value 1 and 0 for transmitting message successfully to sink node or not. The optimal selective method is chosen for maximizing total reward.

In the overview of section 2 we will explain about related work on selective forwarded method for energy efficiency, in section 3 explain problem definition, in section 4 describe proposed work and model of sensor nodes, in section 5 shows the experimental analysis and finally in section 6 define conclusion and future work.

\section{RELATED WORK}

The IDEALS algorithm [10] built under the concept of message priorities as well as power, which tries to make important messages for discarding all messages except those which is high importance when battery resources are scarce, by using this method tries to increase the network lifetime. On paper [8] proposed that energy efficient aware by Bayesian model which based on learning pattern to establish intelligent decisions. The sensor node reads the path from past routing decision with parameter available energy and message importance i.e. transmission belongs to either success or failure.

Every node decides while neighbor node forwarded message for improvement of routing by probabilistic calculation. By using probability estimations reduce the energy of each node in the network, two models taken i.e. within geographic location and other outside from geographic location. By using learning phase sensor node do not need to exchange any specific information among nodes to carry out; it just uses the information for forwarded transmission. A drawback in this model is that energy efficient according to geographic location under coverage area sensor node transmits messages within a limited area and also this probabilistic method was easily simplified for theoretical not practically.

In paper [5] a new energy efficient method are proposed which based on the selective forwarded method. The result of this proposed system increases the performance of the global importance of message by the destination node. In selective forwarded recursive method consider important distribution but non parametric estimation does not depend on it, so the procedure require storing all importance value each time, In this method increases global performance if assign local correlation then it affect on system, but drawback was that, not optimal for practically because of mathematical formulations i.e. Newton Rapson method. In this paper compared selective and non selective forwarded method and from that only highest priority message are arriving to sink node , Estimated $Q_{k}$ by using exponential , Pareto, uniform distribution for finding out maximum like hood function to obtain optimal energy and got high performance. In this method require less complex calculation for efficient energy.

In this paper [11] an optimal selective transmission under energy constraints in sensor networks with different importance, energy cost, the available battery and statically distribution. There are 3 directions for find out the optimal solutions. Optimal selective transmission method are used in some theorems, first theorem was statistically independent sequence second one assume stationary with distribution function, third consider selective transmitter with constant threshold and forth theorem considered only threshold function with those theorem compared with sensor network and isolated network and find out the best performance and optimal efficient energy but comparison are not optimum as well as no delay analysis method was used for that used different method in further work for better efficient energy. Maximizing the lifetime of system for efficient energy given in [12].

In paper [13] proposed stochastic tools are used to develop a selective message forwarding scheme for 3 different cases. It describes the Markov decision process method for

finding the optimal solutions from three theorems, the first one used for a sequence procedure with backward recursive but no optimal solution find from it, second theorem is used stationary method in that each node are not possible to move randomly at different place so that this theorem has no optimal solution for energy efficient. The third theorem considered the constant threshold from this method get the expected reward and efficient energy to optimal solution. Some of the energy strategy methods are already defined in [14] .These three theorems compare with each other and find 
out third one is performed best for network lifetime and the first one is the worse but less signaling overhead.

\section{PROBLEM DEFINATION}

Due to the constraints in Wireless sensor networks such as bandwidth, lifetime of battery, speed of the processor (CPU) and amount of memory there is an essential need for effective communication techniques for improvement of quality of collected data. Routing protocols and stochastic tools from this perspective have a very important role in wireless sensor networks. In existing system learning method are applied for saving energy with some mathematical formulation but it was complex computation and took long time for finding out, then next find out selective forwarder as a intermediate node for transmitting messages from source to sink and by mathematical formulation took efficient energy but with no delay and unstable network. However, optimal selective forwarder used with a Markov decision process for getting efficient energy level, but which was in locally not globally. The network consists global analysis for increases performance level and prolong the network lifetime. The comparison between adaptive selective forwarded and localglobal forwarder method for making better performance, which based on the constant threshold value and system model makes efficient energy for each node and get optimal result.

\section{PROPOSED WORK}

\subsection{Architecture of System Model}

This paper is implementing self constrained environment network design. In the architecture of the system model describes selective forwarded schemes through which save the energy and make prolong the network. There are different method involves in selective forwarder such as an adaptive forwarder method, local and global forwarder method. The optimal selective forwarded method is leading to expression for an optimal solution. The optimal policy depends upon the message and energy routed to the network by using a routing protocol such as AODV which is very efficient for sensor network, because the advantage of AODV in proposed work is route are maintained with time based state. It is very easily accessible for simulation in network as compared other routing protocol.

There are different channels are used to rout the information with energy from one source to the different source by using Markov decision processes, the level of energy also changes according to time, MDP is a stochastic tool and assign proper delay that used for mathematical implementation for wireless sensor network. Therefore, to use probabilistic method and find out the optimal threshold value which gets more efficient energy as compared learning rules. Finally calculate the battery power and comparison between adaptive forwarder and local-global forwarder for getting more optimal saving energy and stable network, which was not happening in previous.

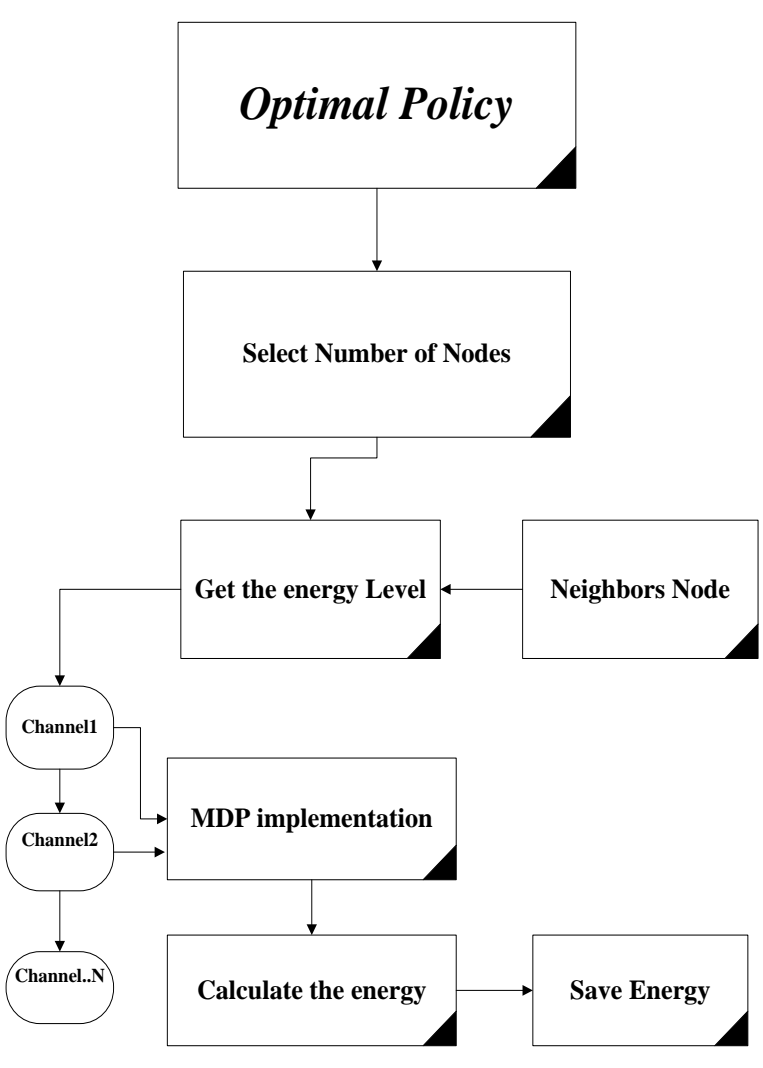

Fig 1: Architecture of the system model

\subsection{Sensor Model}

Sensor node is a collection of nodes are defined as $\mathrm{n}$, one of the nodes selects as a source node and another node select as a sink node. For transmission of messages from source to destination which considered how much energy are available on that node .Energy consist two types such as available energy $\left(a_{k}\right)$, external energy $\left(s_{k}\right)$.The policy is used to take the proper decision whether the node transmitted message successfully or discarded which are $d_{k}\left(a_{k}, z_{k}\right)$.The decision assigns the value 0 or 1 and the action are defending that decision. Energy at time k can be expressed recursively as [9]

$$
a_{k}+1=a_{k} \cdot d_{k} E 1\left(s_{k}\right)-\left(1-d_{k}\right) E 0\left(s_{k}\right)
$$

$E 1\left(s_{k}\right)$ Energy consumed when the node decides to transmit the message, $E 0\left(s_{k}\right)$ is the energy consumed when the message is discarded. According to external energy level increases the probabilities and successful message are also increasing. The probability of any state transition from $\mathrm{k}$ to $\mathrm{k}+1$, to find $p\left(s_{k}+1 \mid s_{k}, d_{k}\right)$ given that at time $\mathrm{k}$ the state was $s_{k}$ and the decision made $d_{k}$. The energy consumed during time $\mathrm{k}+1$ is $a_{k-} a_{k}+1$ and that the energy dynamics are given by (1), the transition probability $p\left(s_{k+1} \mid s_{k}, d_{k}\right)$ can be

$\mathrm{p}\left(\mathrm{s}_{\mathrm{k}}+1 \mid \mathrm{s}_{\mathrm{k}}, \mathrm{d}_{\mathrm{k}}\right)=\left(\mathrm{d}_{\mathrm{k}} \mathrm{p} 1, \mathrm{k}\left(\mathrm{a}_{\mathrm{k}}-\mathrm{a}_{\mathrm{k}}+1 \mid \mathrm{z}_{\mathrm{k}}+1\right)+(1-\right.$
$\left.\left.\mathrm{d}_{\mathrm{k}}\right) \mathrm{p} 0,\left(\mathrm{a}_{\mathrm{k},} \mathrm{a}_{\mathrm{k}}+1 \mid \mathrm{z}_{\mathrm{k}}+1\right)\right) \mathrm{p}_{\mathrm{k}}+1\left(\mathrm{z}_{\mathrm{k}}+1\right)$

Finally find out reward functions means which success index is used, there are 3 success indexes such as a global success index, Local success index, Zero success index. The reward at time $\mathrm{k}$ will be

$$
r_{k}=s_{k} q_{k} u\left(a_{k}-E 1, k\right)
$$


The total reward up to time $\mathrm{k}$ is defined in [13]

$$
\mathrm{t}_{\mathrm{k}}=\sum_{\mathrm{i}=0}^{\mathrm{k}} \operatorname{diri}=\sum_{\mathrm{i}=0}^{\mathrm{k}} \operatorname{diqisku}(\mathrm{ai}-\mathrm{E} 1, \mathrm{i})
$$

The selection forwarding policy is chosen in order to maximize the total expected reward defined as

$E\left\{t_{\infty}\right\}=E\left\{\lim _{k \rightarrow \infty} t_{k}\right\}$

\subsection{Optimal Selective Forwarded}

The number of states is assigned from source to destination, the number of decision or action $\mathrm{A}(0,1)$ depend on transmission of the message ,The transition probability are assigned for any state to another state. The reward functions assign to measure the success of transmission. The action defines finite means using Markova process, which is optimal due to depend on the states. To find out the optimal selective forwarded method proposed theorem.

Theorem: The available energy a, $Q(a, z)=1$, The optimal threshold converts to a constant value and grow linearly expected behavior. Optimal threshold is the difference between two expected rewards. This situation can be observed when $Q(a, z)$ does not depend on a. It related to one equation if $\lim _{a \rightarrow \infty} \mu(a, z)$ exits, it must be equal tolim $\mu(a, z)=$ $(\mathrm{E}\{\mathrm{E} 1 / \mathrm{z}\}-\mathrm{E}\{\mathrm{E} 0 / \mathrm{z}\}) \tau$, this equation is based on adaptive procedure.

\subsection{Constant Energy Threshold}

For comparative purpose derived some expression in the selective forwarded method based on constant threshold, where $\mu_{\mathrm{c}}$ is a constant threshold .If $\mu_{\mathrm{c}}=0$ the constant threshold transmitter reduce to non selective transmitter for that different formulation are calculated. The threshold defines constant value and assigns reward to grow linearly. Basically the optimal threshold is used for difference between two expected rewards, this value are used for both behaviors in the case of stationary as well as in asymptotic .In proposing a system constant threshold assigns for deciding the node those are enter for routing in the network. Let us assume that node decision is given by

$$
d_{k}=u\left(s_{k}-\mu_{c}\right) u\left(a_{k}-E\left(s_{k}\right)\right.
$$

A constant forwarding threshold that did not change a long time and involving asymptotic optimally. For using this method get efficient energy within network and performance are also increasing. The theorem was described in given [13] proposed work, where $\tau$ is a solution of

$\{c 0\} \tau=\{((z)-(\{c 1 \mid z\}-\{c 0 \mid z\}))+$

Parameter $\tau$ can be estimated in real time based on the available information at the time $:\{(\mathrm{z} \ell, q \ell), \ell=0, \ldots, k\}$.

Defining the mean energy difference

$\Delta(z)=E\{c 1 \mid z\}-E\{c 0 \mid z\}$

If the mean energy difference, $\Delta(\mathrm{z})$ is unknown, it can be estimated from data. Making the simplifying assumption that the energy cost does not depend on $\mathrm{z}$ (which may be realistic, for instance, if $\mathrm{z}$ only contains the importance value), it can be estimated as the difference of the average costs of previous decisions. [13]

\subsection{Estimate Success Index}

A simple estimate of the selective communication policy $\left(\mathrm{a}_{\mathrm{k}}, \mathrm{z}_{\mathrm{k}}\right)=\left\{\left(\mathrm{a}_{\mathrm{k}}-\mathrm{c} 1\right) \mid \mathrm{a}_{\mathrm{k}}, \mathrm{z}_{\mathrm{k}}\right\}$ can be derived by assuming that: a) it does not depend on $a_{k}$ (i.e., the subsequent forward/discard decision taken at the receiving node is independent of the energy state at the transmitting node), and b) each node can know about the success of the transmission. When $q_{k}$ represents the local success index, each node is able to listen to the retransmission of a message that has been previously sent (i.e. each node can observe $q_{k}$ when $d_{k}=1$ ) [9]. The optimal selective policy is optimized by the global performance which can be obtained from the theorem.

\section{EXPERIMENT AND ANALYSIS}

In this section analyze performance through different methods of selective forwarder by simulation based on NS-2.34.

a) Take 40 nodes as a sensor node from that some take as a source node, some of as a adaptive forwarder node and one node consider as a sink node. Initially $a_{k}$ and $s_{k}$ consider idle.

b) The threshold constant value assigns for maximizing packet distributions.

c) AODV routing protocol is used for routing packets in network, retransmission and importance of message decided.

d) The energy level changes according the estimate $Q_{k}$ time, which depend on communication, transmission, receptions. The energy level changes when energy of each node reduces.

e) Calculate the energy level of the battery and the degree of probability for battery.

f) Compare adaptive selective forwarder and local-global index forwarder method shows locally-global index is saving energy.

g) As Compare both methods get optimum energy for increasing the performance of the network as well as increasing the lifetime of the network.

During the simulation of Adaptive forwarder some parameters are calculated such as an end to end delay, throughput and packet delivery. The throughput is calculated by using start time and stop time such as start time $=0.40$ and stop time $=5.28$.

Generated packet $=16$

The total dropped packet $=6$

Average end to end delay $=24.7077 \mathrm{~ms}$

Average throughput $[\mathrm{kbps}]=226.17$ 


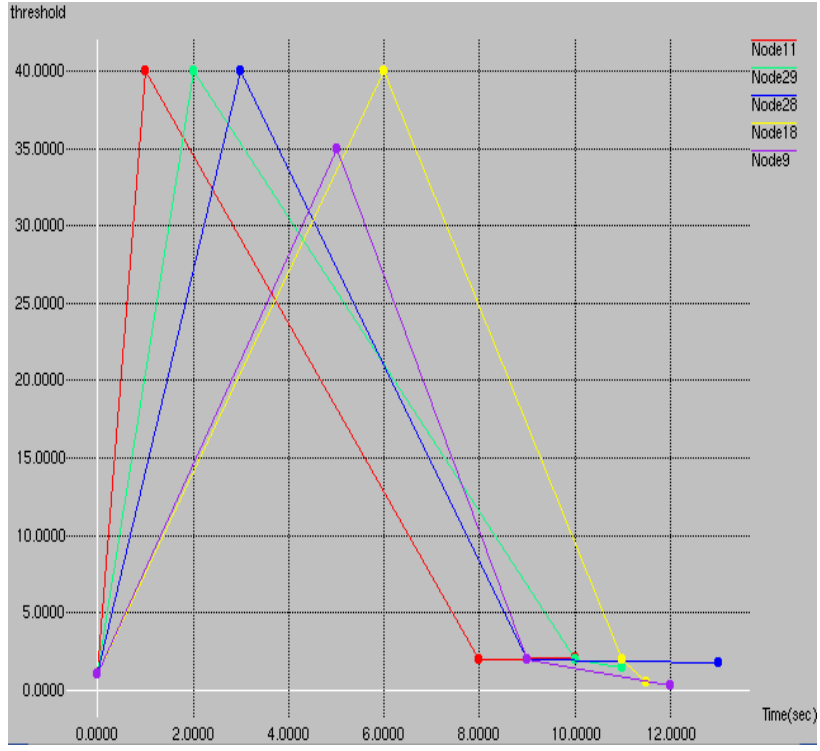

Fig 2: Threshold for packet distribution (Energy-Joules)

In Fig. 2 shows the constant threshold value corresponding number of nodes, the packet distributed according to the threshold .Take a constant threshold and consider those nodes are on constant threshold that are only ready for routing in the network such as node 11, 29, 28 and 18. Node 9 has no permission to routed network due to below of constant threshold. The constant threshold value considered because of transmission of packets successfully to sink node with maximum.

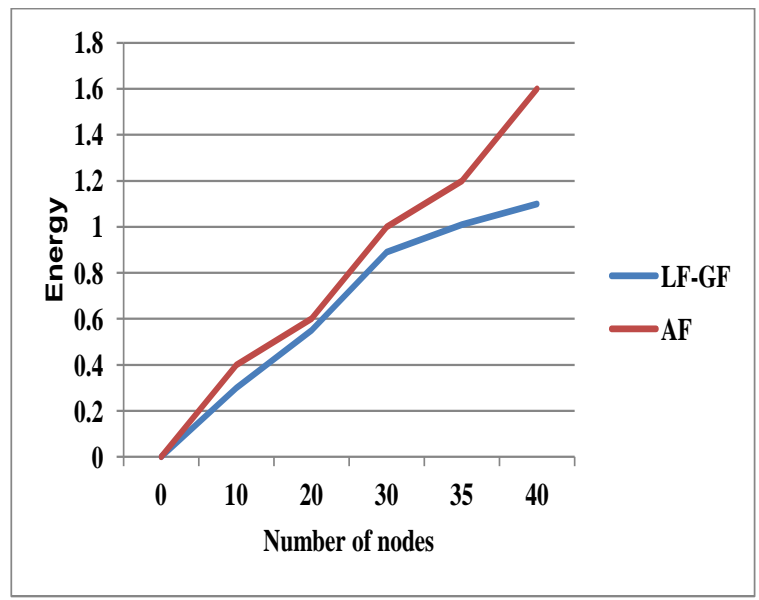

Fig 3: Comparison between adaptive and local-global selective forwarding policies method.

In Fig. 3 shows $\mathrm{x}$-axis consist number of nodes and y axis consist energy gain in the form of a joule. The result of analysis for 40 sensor nodes, as shown in Fig. 3 LF-GF is 1.1 energy gains and AT is 1.6 energy gains. So, as compare AT selective forwarder method local with global forwarder method are saved maximum energy in the network

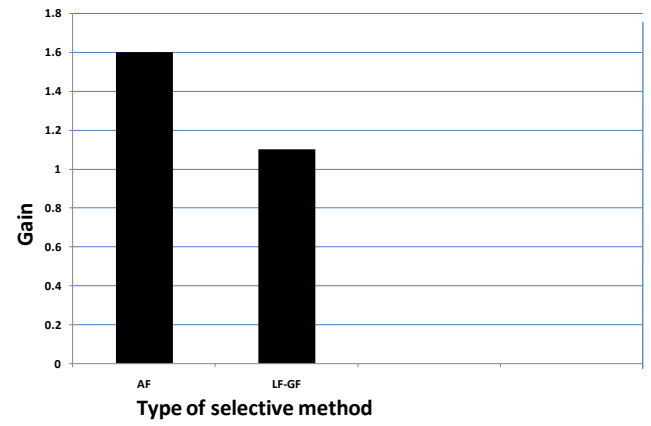

Fig 4: Energy gain performance between adaptive and local-global method

In summary, the gain value increases of the LF-GF is the behavior of Local-global selective sensor networks are energy-efficient compared with the adaptive selective one, allowing the formers to obtain a huge increase in network lifetime at the same time that maximizes the communication efficiency up to the sink node, and therefore, notably improves the global performance. However conclude that local with a global forwarder method is better than selective methods.

\section{CONCLUSION AND FUTURE WORK}

In this paper presented a selective forwarder method with different classification and applying this method with Markov decision process. By using MDP, easily find out optimal energy for large sensor networks as well as easily find out battery level which calculated by probabilistic formulation. Finally from simulation and analysis get the efficient energy level between adaptive selective method and local-global forwarded method. The energy level is increased in localglobal forwarder and get maximum selective by using a constant threshold.

This method has a strong theoretical component results from a practical point of view. Because not only it can provide basic information for the design of future system but also the developmental scheme can be included in many existing routing protocols. Therefore future work includes the implement in some kind of estimation protocol also considered the ideal value for future enhancement.

\section{ACKNOWLEDGMENT}

The authors would like to thank the anonymous reviewers for their detailed, valuable comments and constructive suggestion.

\section{REFERENCES}

[1] Xu N.,"A Survey of Sensor Network Applications", IEEE Communications Magazine, vol. 40, 2002.

[2] Akyildiz I. F., Su W., Sankarasubramaniam Y., and Cayirci E., "A survey on sensor networks," IEEE Commun. Mag., vol. 40, no. 8, pp. 102-114, Aug. 2002.

[3] S.El ashheb A., "Performance Evaluation of AODV and DSDV Routing Protocol," Internationational Conference 
on Computer Network and Communication System. IPCSIT, vol.35 (2012) IACSIT Press.

[4] Manoj B. S., Siva Ram Murthy S., "Ad Hoc Wireless Networks Architectures and Protocols", Pearson, 2004.

[5] Arroyo-Valles R., Marques A. G., and Cid-Sueiro J., "Energy-efficient selective forwarding for sensor networks," in Proc. Workshop on Energy in Wireless Sensor Networks (WEWSN'08), in conjunction with DCOSS'08, June 2008.

[6] Munir A. and Gordon-Ross A., "An MDP-based application oriented optimal policy for wireless sensor networks," in Proc. 7th IEEE/ACM Int'l Conf. on Hardware/Software Codesign and System Synthesis (CODES+ISSS ’09), pp. 183-192.

[7] Puterman M., "Markov Decision Processes", WileyInterscience, 2005.

[8] Arroyo-Valles R, Marque A. Gs, Vinagre-D'iaz J, and Cid-Sueiro J, "A Bayesian decision model for intelligent routing in sensor networks," in Proc. 3rd IEEE Int'l Symp. on Wireless Comm. Systems (ISWCS '06), Sep. 2006.
[9] Arroyo-Valles R, Marques A. G, and Cid-Sueiro J., Wireless Sensor Networks. IN-TECH ch. Energy-aware Selective Communications in Sensor Networks. 2010.

[10] Merrett, G., Al-Hashimi, B., White, N. \& Harris, N Information Managed Wireless Sensor Networks with Energy Aware Nodes, Proc. NSTI Nanotechnology Conf. and Trade Show (NanoTech '05), pp. 367-370. 2005.

[11] Arroyo-Valles R., Marques A. G, and Cid-Sueiro J., "Optimal selective transmission under energy constraints in sensor networks," IEEE Trans. Mobile Computing, vol. 8, no. 11, pp. 1524-1538, Nov. 2009.

[12] Alferia A, Bianco A, Brandimarte P, Chiasserini C. F, "Maximizing system lifetime in wireless sensor networks," European Journal of Operational Research 181 , pp.390-402, 2007.

[13] Arroyo-Valles R, Marques A. G, and Cid-Sueiro J, "Optimal Selective Forwarding for Energy Saving in Wireless sensor networks," IEEE Trans. Mobile Computing, vol. 10, no. 1,pp. 1536-1276, Jan. 2011.

[14] Van Phan C, Park Y, Hyun Choi H, Cho J and Geun Kim J, "An Efficient Transmission Strategy for Wireless Sensor Network," IEEE Transactions on Consumer Electronics, Vol. 56, No. 2, May 2010 